\title{
Intraocular foreign body embedded in ciliary body
}

\author{
Kuan-Jen Chen*, Chun-Hsiu Liu and Laura Liu \\ Department of Ophthalmology, Chang Gung Memorial Hospital and College of Medicine, Chang Gung University, Taoyuan, Taiwan
}

\begin{abstract}
A 34-year-old man complained that his right eye was struck by a metal object. His visual acuity was $12 / 20$. Slit-lamp biomicroscopic examination showed a peripheral corneal full-thickness laceration. Bone-window computed tomography revealed a high-reflectivity metallic foreign body in the angle area of the right eye. Ultrasonic biomicroscopic examination displayed a hyperreflective intraocular foreign body (IOFB) in the ciliary area. The IOFB was externally removed by creating a limbusbased scleral flap. One month later, the vision was 20/20 without complications.
\end{abstract}

\section{Introduction}

Intraocular foreign bodies (IOFBs) vary in presentation, outcome, and prognosis [1]. Localization and surgical removal of an IOFB are perhaps the most unpredictable, and they require intense preoperative evaluation and patient counseling. The difficulty of surgical IOFB removal depends on the size and location of the IOFB. The technique and instrument for IOFB extraction in the ciliary area are rare [2-4]. Although the internal removal of IOFBs in the ciliary area is the main strategy employed in modern techniques [2-4], this can result in complications, such as cataract, internal bleeding with angle damage, and glaucoma. We describe several imaging systems and a technique for successful external removal of an IOFB in the ciliary area after the failure of internal extraction.

\section{Case report}

A 34-year-old man presented at the emergency department of the Chang Gung Memorial Hospital, Taoyuan, Taiwan complaining that his right eye was struck immediately after he pounded a metal object with a metal chisel. He had no history of trauma to the eye or previous surgery. His vision was $12 / 20$ and 20/20 in the right and left eyes, respectively. Intraocular pressure in the right and left eyes was $17 \mathrm{mmHg}$ and $16 \mathrm{mmHg}$, respectively. On slit-lamp biomicroscopic examination, an obliquely peripheral corneal 1-mm Seidel-negative full-thickness laceration was identified at 4 oclock position. The anterior chamber formed a $1+$ cell, and no hypopyon or hyphema were observed. The edge of what appeared to be a metal fragment was invisible in the angle of the anterior chamber. The lens was clear, and fundus had no abnormality. Computed tomography (CT) revealed a high-reflectivity metallic foreign body in the angle area of the right eye (Figures $1 \mathrm{a}$ and $1 \mathrm{~b}$ ).

The patient underwent IOFB removal from the anterior chamber under general anesthesia. Limbal paracentesis was performed at 11 oclock position. An internal magnet was inserted around the angle to remove the IOFB, but this procedure had little success. The IOFB was not extracted, and the intraocular magnet damaged the angle causing hemorrhage. The wound was sutured with 10-0 nylon. Because of the risk of infectious endophthalmitis, intravitreal vancomycin and ceftazidime injections were administered. One day later, slit-lamp biomicroscopic examination revealed mild hyphema and lenticular vacuoles (Figure 1c) as well as no wound leakage. Ultrasonic biomicroscopic examination displayed a hyperreflective foreign body in the ciliary area (Figure 1d). Four days after the injury, retrobulbar anesthesia was administered. The conjunctiva was incised around the lower third of limbus adjacent to the nasal corneal wound. A rectangular limbusbased scleral flap was created near the corneal laceration (Figure 1e). The sclera and uvea were separated using a knife and extended to the limbus, and the IOFB was localized with a Kuhn intraocular magnet (Geuder, Germany). After splitting the ciliary tissue, the IOFB was removed successfully (Figure 1f). Little hemorrhage was observed during the surgical procedure. The scleral flap and conjunctival periotomy were sutured. One month later, the patient had a white eye with no sign of flap bulging. Lens and fundus revealed no abnormalities, and the vision was 20/20 without complications.

\section{Discussion}

Although IOFBs can typically be easily visualized, visualizing them may be difficult in eyes with ciliary foreign bodies. Several imaging systems are available for detecting IOFBs, such as CT, ocular B-scan ultrasonography, ultrasonic biomicroscopy (UBM), and anterior segment optical coherence tomography (AS-OCT) [2,5-9]. Noncontrast CT not only reveals the IOFB location but also provides information regarding the IOFB dimensions, thus enabling the surgeon to determine the most optimal extraction route $[5,7,9]$. However, foreign bodies in the posterior iris and ciliary body are a considerable problem regarding localization using B-scan ultrasonography or CT. Soft-tissue window orbital CT scans usually overestimates the size of the metallic foreign body and fails to reveal the exact IOFB location, which is misdiagnosed in intraand extraocular regions (Figure 1a). Bone-window orbital CT scans not only minifies the metallic IOFB size but also reveals the exact IOFB location (Figure 1b). UBM and AS-OCT facilitate obtaining in-depth

${ }^{*}$ Correspondence to: Kuan-Jen Chen, Department of Ophthalmology, Chang Gung Memorial Hospital, 5 Fuhsing Street, Kwei-Shan, 333, Taoyuan, Taiwan, Tel: 886-33281200 ext. 8671, Fax: 886-3-328-7798, E-mail: cgr999chiayi@yahoo.com.tw

Key words: bone-window orbital computed tomography, ciliary body, intraocular foreign body, ultrasonic biomicroscopy

Received: October 05, 2018; Accepted: October 19, 2018; Published: October 23, 2018 


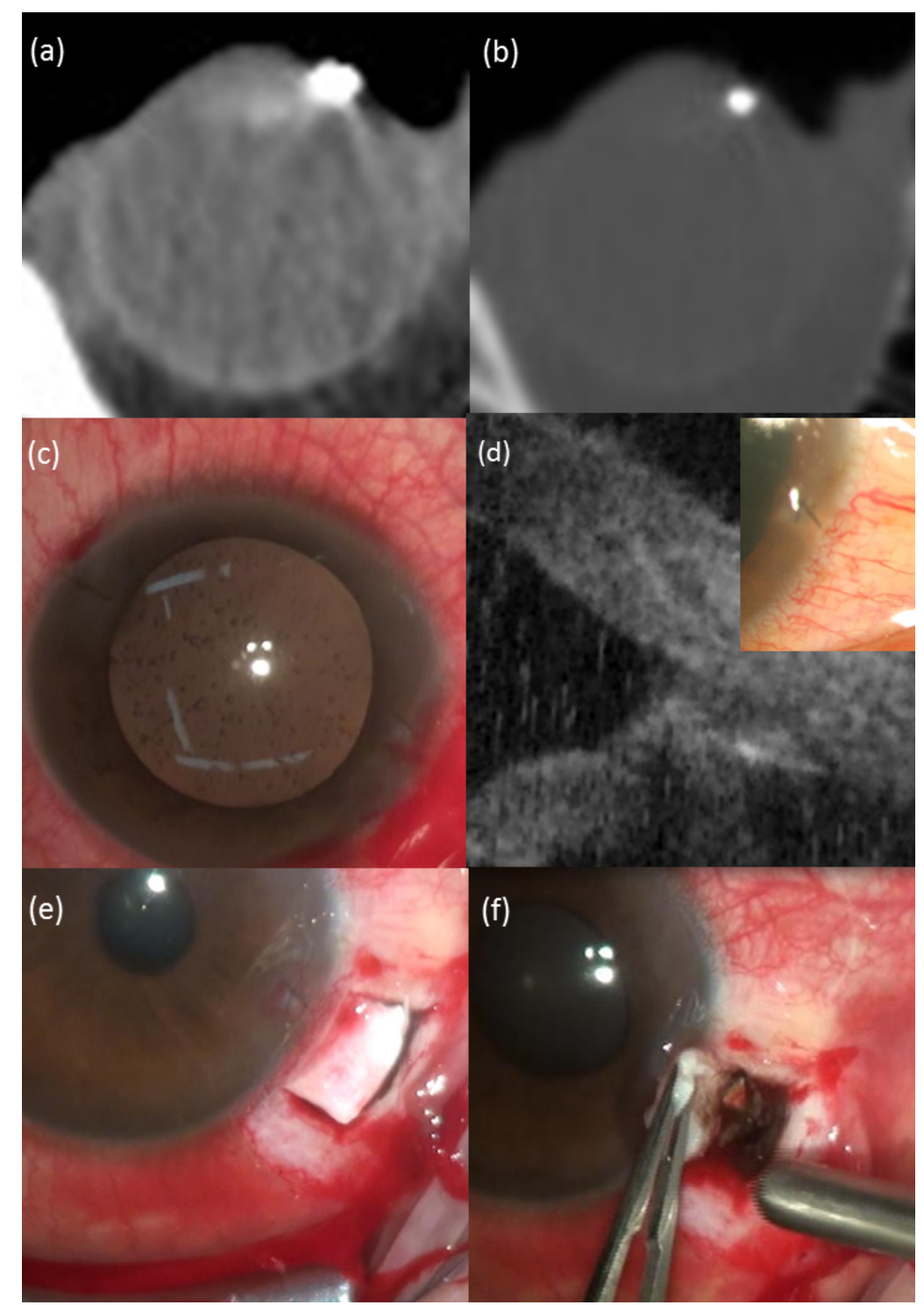

Figure 1. A soft-tissue window orbital computed tomography (CT) scan revealing a metallic foreign body resting on the wall of the eye. (a) A bone-window orbital CT scan reveals an intraocular metallic foreign body. (b) Twenty-four hours after the failure to remove the foreign body, a slit-lamp photograph of the right eye reveals the repaired corneal laceration and vacuoles in the anterior lenticular cortex. (c) An ultrasound biomicroscopy scan reveals a hyperechogenic structure with back-shadowing embedded in the ciliary body. (d) An intraoperative photograph of a limbus-based scleral flap adjacent to the corneal laceration. (e) A triangular metallic foreign body embedded in the ciliary body. (f) After splitting the ciliary tissue, the IOFB is successfully removed using a Kuhn intraocular magnet. The foreign body was $1.2 \mathrm{~mm}$ in length and $1 \mathrm{~mm}$ in width

information regarding IOFBs in the anterior segment $[2,6,8,10]$. UBM and AS-OCT are safe, noninvasive imaging tools for localizing occult IOFBs located in the anterior segment and may be the only means of accurately localizing these IOFBs $[2,6,8,10]$. Moreover, AS-OCT is superior to UBM for imaging small lesions of the anterior iris, but the UBM is suitable for imaging larger iris lesions with posterior or ciliary body extension [10].

IOFB extraction is a critical procedure because it is associated with many potential complications. After performing a comprehensive workup and determining the IOFB type, surgeons should emphasize the extraction wound and determine the least traumatic method for treating the ocular tissue. Previously, foreign bodies of the ciliary area were removed through the anterior chamber or through endoscopic vitrectomy [2-4]. However, these methods created complications such as cataract, internal bleeding with angle damage, and glaucoma. When a foreign body is deeply embedded in the ciliary body, the metallic for- eign body is often entangled in the ciliary muscle and can usually be extracted safely with gentle maneuvering by using a knife and the magnet itself. The extracted wound can be a limbus-based scleral flap or a direct limbal incision. Limbal incision with IOFB extraction is associated with the risk of iris protrusion and iridodialysis. A limbus-based scleral flap with IOFB extraction not only reduces these problems but also avoids traumatic cataract and hyphema when approached through the anterior chamber.

In summary, we describe several imaging systems and a technique for external removal of an IOFB in the ciliary area that may enable surgeons to avoid the internal approach, which generates complications such as hemorrhage, cataract, and glaucoma. Bone-window orbital CT scans and UBM are better imaging tools for localizing occult metallic IOFBs located in the ciliary body. This technique of creating a limbusbased scleral flap may be particularly useful in extracting IOFBs in the ciliary area. 


\section{References}

1. Ehlers JP, Kunimoto DY, Ittoop S, Maguire JI, Ho AC, et al. (2008) Metallic intraocular foreign bodies: characteristics, interventions, and prognostic factors for visual outcome and globe survival. Am J Ophthalmol 146: 427-433. [Crossref]

2. Kaushik S, Ichhpujani P, Ramasubramanian A, Pandav SS (2008) Occult intraocular foreign body: ultrasound biomicroscopy holds the key. Int Ophthalmol 28: 71-73. [Crossref]

3. Marra KV, Yonekawa Y, Papakostas TD, Arroyo JG (2013) Indications and techniques of endoscope assisted vitrectomy. J Ophthalmic Vis Res 8: 282-290. [Crossref]

4. Wong SC, Lee TC, Heier JS, Ho AC (2014) Endoscopic vitrectomy. Curr Opin Ophthalmol 25: 195-206. [Crossref]

5. Dass AB, Ferrone PJ, Chu YR, Esposito M, Gray L (2001) Sensitivity of spiral computed tomography scanning for detecting intraocular foreign bodies. Ophthalmology 108: 2326-2328. [Crossref]
6. Deramo VA, Shaw GK, Baumal CR, Fineman MS, Corrêa ZM, et al. (1999) Ultrasound biomicroscopy as a tool for detecting and localizing occult foreign bodies after ocular trauma. Ophthalmology 106: 301-305. [Crossref]

7. Lakits A, Prokesch R, Scholda C, Bankier A (1999) Orbital helical computed tomography in the diagnosis and management of eye trauma. Ophthalmology 106: 2330-2335. [Crossref]

8. Wylegala E, Dobrowolski D, Nowi̊̊,,ska A, Tarnawska D (2009) Anterior segment optical coherence tomography in eye injuries. Graefes Arch Clin Exp Ophthalmol 247: 451-455. [Crossref]

9. Zinreich SJ, Miller NR, Aguayo JB, Quinn C, Hadfield R, et al. (1986) Computed tomographic three-dimensional localization and compositional evaluation of intraocular and orbital foreign bodies. Arch Ophthalmol 104: 1477-1482. [Crossref]

10. Wong SC, Lee TC, Heier JS, Ho AC (2014) Endoscopic vitrectomy. Curr Opin Ophthalmol 25: 195-206. [Crossref]

Copyright: $₫ 2018$ Chen K. This is an open-access article distributed under the terms of the Creative Commons Attribution License, which permits unrestricted use, distribution, and reproduction in any medium, provided the original author and source are credited. 